\title{
Popping Properties of Rice as Influenced by Cultivars, Temperature and Time of Popping
}

\author{
Khaled M.H. Abd El Salam ${ }^{1}$, Ahmed M.Shaalan ${ }^{2}$ and Medhat A.E. El-Dalil ${ }^{1}$
}

\begin{abstract}
This research aimed to determine the effect of processing conditions, including temperature and time of popping on expansion of popped rice cultivars. The results of the two seasons 2013 and 2014 showed significant differences between cultivars for most studied characters. Giza 178 rice cultivar showed highest significant values for expansion ratio, weight of popped and popping percentage in both seasons. Increasing temperature degree from 220 to $260{ }^{\circ} \mathrm{C}$ significantly increased all studied characters in both seasons. In addition, the optimum time for all studied characters was 60 seconds. Interaction for expansion ratio between 40 and 60 second, at $260^{\circ} \mathrm{C}$, revealed a gradual increase but with different magnitude for each cultivar.
\end{abstract}

Keywords: Rice, temperature, time, expansion ratio and popping percentage.

\section{INTRODUCTION}

The snack food is one of the most important areas of the food industry. Designing snack foods today can be a complex process to meet changing consumers taste and expectations and elusive search for something unique that also appeals to a wide variety of people. Most snack manufacturers use some forms of existing technology as the basis for creating snack products and incorporate variations that increase the resulting snacks' health image. Therefore, popping using advance technologies are processes which can accomplish all these targets. As a simple, inexpensive and quick traditional method of dry heat application for preparation of weaning food formulations and ready to eat snacks products, popping have been practiced since hundreds of years.

Popped rice is one of the earliest known rice based popular traditional ready to eat breakfast cereal products in South-East Asia (Bhat Upadya et al., 2008). The whole grain produce contributes numerous beneficial nutrients for human health including dietary, fiber, vitamins, minerals and phytochemicals (Maisont \& Narkrugsa, 2010).

Expansion value is the most important quality parameter for popped rice. Proper moisture content is known to govern expansion value as it creates the necessary vapour pressure inside the kernels before popping. Moisture loss decreases popping performance (Song and Eckhoff, 1994), whereas excess moisture produces low popped volume (Shimoni et al., 2002). Except moisture, not much is known on the factors influencing popping in rice unlike in maize, where several physico-chemical properties of the kernel are well studied (Dofing et al., 1990; Mohamed et al., 1993; Tian et al., 2001; Ceylan and Karababa, 2002).

To avoid the limitations of conventional popping methods, electromagnetic waves such as microwaves are used nowadays, which provides better energy efficiency in very short time. Microwave energy is worldwide used for producing popcorn. Though a wide range of cereals are used for popping, only few of them pop well. That may be due to the factors which influence popping qualities of cereals, such as season, varietal differences, grain characters, i.e., bran content, bran thickness, moisture content, type of endosperm, physical characters of grains and also the method of popping (Hoke et al., 2005; Mirza et al., 2014; Joshi et al., 2014). High popping could be achieved with paddy containing moisture content between 14 and 15\%. Time of heating is a sensitive parameter for sharp rise in popping percentage as compared to power level (Swarnakar et al., 2014)

Initial micropore size, popping temperature, surface tension, yield stress and rupture stress are some other factors that influence the popping characteristics of the grains (Henry et al., 1995). The kernel properties of cereals, such as size, shape and density may also affect expansion volume (Hoke et al., 2005; Joshi et al., 2014).

Varietal differences may affect popping where Bhat Upadya et al. (2008), found that Intan recorded the highest popping percentage (49\%) followed by Putta Bhatta, Medhini Sanna Bhatta and IR64. They also reported that zinc and amylose content had significantly negative correlation with pop ability. Abd El Salam (2006), found that the optimum conditions for producing popped rice with best yield were Giza 178 variety with $14 \%$ moisture content, $300{ }^{\circ} \mathrm{C}$ heating temperature, one minute time and 50 grams of paddy rice.

\footnotetext{
${ }^{1}$ Rice Technology Training Center (RTTC), Field Crops

Research Institute, Agricultural Research Center, Alexandria, Egypt.

${ }^{2}$ Faculty of Desert and Environmental Agriculture, Matrouh Branch, Alexandria University.

Received November 26, 2015, Accepted December10, 2015
} 
The present study was conducted to determine the effect of processing conditions, including temperature and heating time on expansion of popped rice cultivars.

\section{MATERIALS AND METHODS}

Two experiments were performed at Grain Quality Labs; Rice Technology Training Center (RTTC), Alexandria, Egypt, to determine the effect of temperature, time and their interaction on popping expansion of rice cultivars. Newly harvested certified seeds in 2013 and 2014 growing seasons of five rice cultivars namely, Sakha 102, Sakha 106, Giza 178, Giza 182 and Egyptian Yasmin were provided by Rice Research Program, Field Crop Research Institute, Agriculture Research Center, Sakha Kafr, El-Sheikh, Egypt. A split-split plot design with three replicates was used in both seasons. The main plots were devoted to rice cultivars and the sub plots were occupied by five temperature levels $\left(220,230,240,250\right.$ and $\left.260{ }^{\circ} \mathrm{C}\right)$ whereas, the sub-sub plots were assigned to four time intervals $(40,50,60$ and $70 \mathrm{sec})$. The characters were measured on $14 \%$ moisture content basis and fixed sample weight for popping was 50 gram.

The studied popping characters were expansion ratio, weight after popping (excluding loss in moisture), density $\left(\mathrm{g} / \mathrm{cm}^{3}\right)$, weight of popped rice $(\mathrm{g})$ and popping percentage $(\%)$.

Popped and unpopped grains were separated using a USA standard testing sieve (No. 6 Fischer Scientific co. Pitsburgh, PA). The popping percentage was calculated as mentioned by Swarnakar et al. (2014) as follows: Popping $\%=$ weight of popped kernels / weight after popping $X 100$. Expansion ratio was the ratio of the volume of the popped kernels without the husk to that of whole brown rice obtained from $25 \mathrm{~g}$ paddy (Murugesan and Bhattacharya, 1989). Density was determined as described by Delost-Lewis et al. (1992).

A local made electrical popping machine was designed and manufactured in order to study the factors affecting the popping conditions. The equipment consisted of stainless steel vessel which had the following physical dimensions : the internal diameter 23 $\mathrm{cm}$, length $12 \mathrm{~cm}$, internal volume $603.2 \mathrm{~cm}^{3}$. The temperature in the popping vessel was monitored with a thermocouple connected to a voltage regulator. To prevent burning during popping or puffing, a stirrer with a fixed speed ( $80 \mathrm{rpm}$ ) was inserted from outside into the popping vessel.

Analysis of variance was carried out according to Gomez and Gomez (1984) using SAS program, version 8.0. Means were compared using least significant difference (LSD) at 0.05 level of probability.

\section{RESULTS AND DISCUSSIONS Popping properties:}

Popping quality is always determined by calculating the expansion ratio. It is defined as ratio of the volume of the popped without husk to that of raw brown rice at a constant weight (Murugesan and Bhattcharya, 1989). Moreover, determinations of Flake size, popping density, hydration power, hardness, whiteness, size and shape of the end product are of significant importance. Expansion ratio as well as other quality indices have been found to depend on many factors, such as moisture content of rice, kernel size, shape and other physical properties of variety or genotype, harvesting and handling practices, drying conditions, kernel damage, kernel structure, amount and distribution of protein, starch composition, popping temperature, popping method, and several other unexplained factors (Srinivas and Desikachar 1973; Gokmen, 2004). However, among all these factors affecting expansion ratio, moisture content is the most critical factor, because it affects the rate and extent of pressure build up in starch granules (Hoseney et al., 1983).

Popping is a simultaneous starch gelatinization and expansion process, during which grains are exposed to high temperatures for a short time. During this process, super heated vapour produced inside the grains by instantaneous heating, cooks the grain and expands the endosperm suddenly breaking out the outer skin.

\section{1- Effect of cultivars:}

Performance of the studied rice cultivars, in both seasons, are presented in tables (1 and 2). Data revealed that there were significant differences between rice cultivars for all studied characters in the two seasons. Giza 178 rice cultivar showed the highest significant values for expansion ratio (8.61 and 8.06), weight of popped (41.60 and $41.31 \mathrm{~g}$ ) and popping \% (90.31 and $90.36 \%$ ) in 2013 and 2014 seasons, respectively. However, it showed the lowest values for density (103.45 and $107.83 \mathrm{~g} / \mathrm{cm} 3$ ) and weight after popping (46.04 and $45.68 \mathrm{~g}$ ) in 2013 and 2014 rice seasons, respectively. Also, Giza 182 rice cultivar showed highest values for density and weight after popping (143.65 and $47.55 \mathrm{~g}$ ), respectively and lowest values for expansion ratio (6.96), weight of popped (37.68 g) and popping \% (79.14\%) in 2013 season. On the other hand, in 2014 season, Giza 182 showed also superiority for density (147.51) and lowest value for expansion ratio (7.56). Sakha 102 rice cultivar showed the lowest values for weight of popped (35.21 g) and popping \% (76.55 $\%)$. Moreover, Sakha 106 rice cultivar was superior in weight after popping (46.83 g) in that season. This might be due to genetic differences between cultivars in 
grain structure and endosperm characteristics. (Abd El Salam 2006).

\section{2- Effect of temperature:}

Increasing temperature from 220 to $260{ }^{\circ} \mathrm{C}$ significantly increased all studied characters in both seasons (Table 1 and 2). The highest values for expansion ratio (8.51 and 8.60), density (133.0 and $139.60 \mathrm{gm} / \mathrm{cm}^{3}$ ), weight after popping (47.48 and 47.02 $\mathrm{g}$ ), weight of popped (42.38 and $42.50 \mathrm{~g}$ ) and popping $\%(89.28$ and $90.36 \%)$ in 2013 and 2014 seasons, respectively, were obtained with $260{ }^{\circ} \mathrm{C}$. However, the lowest values for expansion ratio (6.86 and 6.92), density (106.97 and $\left.103.11 \mathrm{gm} / \mathrm{cm}^{3}\right)$, weight after popping (45.75 and $45.47 \mathrm{~g}$ ), weight of popped (35.15 and $33.89 \mathrm{~g}$ ) and popping \% (76.86 and 74.51\%) in 2013 and 2014 seasons, respectively, were obtained with $220{ }^{\circ} \mathrm{C}$. This might be due to that increasing the heating temperature could accelerate both melting of rice kernels and evaporation of water in rice. The melting renders the rice kernel elastic and expandable whereas, the evaporation exerts the pressure needed for expansion. Therefore, the expansion of rice increased with increasing heating temperature. Similar results was reported by (Hsieh et al., 1989 and Huff et al.,1992).

\section{3- Effect of time:}

Data presented in (Table 1 and 2) showed that the optimum time for all studied characters was 60 seconds. The highest values for expansion ratio (8.04 and 8.29), density (123.10 and $\left.127.63 \mathrm{gm} / \mathrm{cm}^{3}\right)$, weight after popping (47.10 and $46.62 \mathrm{~g}$ ), weight of popped (41.12 and $40.91 \mathrm{~g})$ and popping \% (87.33 and 87.71 $\%$ ) in 2013 and 2014 seasons, respectively, were recorded at 60 seconds. In contrast, the lowest values for expansion ratio (7.03 and 7.06), density (111.81 and $115.14 \mathrm{gm} / \mathrm{cm}^{3}$ ), weight after popping (46.08 and 45.78 $\mathrm{g}$ ), weight of popped (37.49 and $36.23 \mathrm{~g}$ ) and popping $\%(81.29$ and $79.03 \%)$ in 2013 and 2014 seasons, respectively, were obtained at 40 seconds. Increasing time of heating above $60 \mathrm{sec}$ resulted in the scorching, browning of the surface and decreased popping \% (Abd-El Salam 2006).

\section{4- Interaction between cultivars, temperature and time of popping:}

Interaction for expansion ratio between 40 and 60 second, at $260{ }^{\circ} \mathrm{C}$, revealed a gradual increase but with different magnitude for each cultivar. Increase in expansion ratio reached $28.31,16.88,14.61,17.39$ and $20.76 \%$ in the first season, and $25.28,21.41,16.14$, 16.17 and $21.78 \%$ in the second season, for Giza 178, Giza 182, Sakha 102, Sakha 106 and Yasmine, respectively (Table 3).

The other three characters, i.e., weight after popping, weight of popping and popping \% showed similar trend, as in expansion ratio, concerning the magnitude of change in character with increasing heating time from 40 to 60 second (Tables 4, 5 and 6).

Table 1. Mean values for expansion ratio, density $\left(\mathrm{g} / \mathrm{cm}^{3}\right)$, weight after popping $(\mathrm{g})$, weight of popped (g) and popping percentage as affected by cultivars, temperature and time of popping in 2013 season

\begin{tabular}{|c|c|c|c|c|c|}
\hline Treatment & $\begin{array}{c}\text { Expansion } \\
\text { ratio }\end{array}$ & $\begin{array}{l}\text { Density } \\
\left(\mathrm{g} / \mathrm{cm}^{3}\right)\end{array}$ & $\begin{array}{l}\text { Weight after } \\
\text { popping (g) }\end{array}$ & $\begin{array}{l}\text { Weight of } \\
\text { popped (g) }\end{array}$ & Popping (\%) \\
\hline \multicolumn{6}{|l|}{ Cultivars: } \\
\hline G. 178 & 8.61 & 103.45 & 46.04 & 41.60 & 90.31 \\
\hline G. 182 & 6.96 & 143.65 & 47.55 & 37.68 & 79.14 \\
\hline S.102 & 7.10 & 123.27 & 46.82 & 38.01 & 81.08 \\
\hline S.106 & 7.41 & 117.30 & 46.40 & 39.33 & 84.69 \\
\hline Egyptian Yasmin & 7.86 & 110.92 & 46.18 & 41.08 & 88.89 \\
\hline L.S.D 0.05 & 0.10 & 4.04 & $\mathbf{0 . 0 7}$ & 0.10 & 0.35 \\
\hline \multicolumn{6}{|l|}{ Temp ( $\left.{ }^{0} \mathrm{C}\right):$} \\
\hline 220 & 6.86 & 106.97 & 45.75 & 35.15 & 76.86 \\
\hline 230 & 7.11 & 110.29 & 46.17 & 38.06 & 82.48 \\
\hline 240 & 7.54 & 121.43 & 46.61 & 40.55 & 87.02 \\
\hline 250 & 7.93 & 126.89 & 46.97 & 41.54 & 88.48 \\
\hline 260 & 8.51 & 133.00 & 47.48 & 42.38 & 89.28 \\
\hline L.S.D 0.05 & 0.08 & 4.66 & 0.03 & 0.04 & 0.11 \\
\hline \multicolumn{6}{|l|}{ Time (sec): } \\
\hline 40 & 7.03 & 111.81 & 46.08 & 37.49 & 81.29 \\
\hline 50 & 7.51 & 120.86 & 46.47 & 39.17 & 84.26 \\
\hline 60 & 8.04 & 123.10 & 47.10 & 41.12 & 87.33 \\
\hline 70 & 7.77 & 123.00 & 46.73 & 40.38 & 86.42 \\
\hline L.S.D ${ }_{0.05}$ & 0.07 & 3.90 & 0.02 & 0.04 & 0.11 \\
\hline
\end{tabular}


Table 2. Mean values for expansion ratio, density $\left(\mathrm{g} / \mathrm{cm}^{3}\right)$, weight after popping (g), weight of popped (g) and percentage of popped as affected by cultivars, temperature and time of popping in 2014 season

\begin{tabular}{|c|c|c|c|c|c|}
\hline Treatment & $\begin{array}{c}\text { Expansion } \\
\text { ratio } \\
\end{array}$ & $\begin{array}{l}\text { Density } \\
\left(\mathrm{g} / \mathrm{cm}^{3}\right)\end{array}$ & $\begin{array}{l}\text { Weight after } \\
\text { popping (g) }\end{array}$ & $\begin{array}{l}\text { Weight of } \\
\text { popped (g) }\end{array}$ & Popping \% \\
\hline \multicolumn{6}{|l|}{ Cultivars: } \\
\hline G. 178 & 8.06 & 107.83 & 45.68 & 41.31 & 90.36 \\
\hline G. 182 & 7.56 & 147.51 & 46.05 & 38.40 & 83.30 \\
\hline S.102 & 7.66 & 126.26 & 45.94 & 35.21 & 76.55 \\
\hline S.106 & 7.60 & 121.42 & 46.83 & 38.49 & 82.08 \\
\hline Egyptian Yasmin & 8.01 & 113.18 & 46.73 & 40.12 & 85.77 \\
\hline L.S.D ${ }_{0.05}$ & 0.04 & 3.75 & $\mathbf{0 . 0 3}$ & 0.85 & 1.81 \\
\hline \multicolumn{6}{|l|}{ Temp ('C): } \\
\hline 220 & 6.92 & 103.11 & 45.47 & 33.89 & 74.51 \\
\hline 230 & 7.30 & 113.56 & 45.89 & 36.53 & 79.56 \\
\hline 240 & 7.80 & 128.30 & 46.24 & 39.11 & 84.56 \\
\hline 250 & 8.27 & 131.62 & 46.61 & 41.52 & 89.05 \\
\hline 260 & 8.60 & 139.60 & 47.02 & 42.50 & 90.36 \\
\hline L.S.D 0.05 & $\mathbf{0 . 0 3}$ & 3.19 & $\mathbf{0 . 0 3}$ & 0.76 & 1.63 \\
\hline \multicolumn{6}{|l|}{ Time (sec): } \\
\hline 40 & 7.06 & 115.14 & 45.78 & 36.23 & 79.03 \\
\hline 50 & 7.77 & 124.09 & 46.18 & 38.39 & 83.06 \\
\hline 60 & 8.29 & 127.63 & 46.62 & 40.91 & 87.71 \\
\hline 70 & 7.99 & 126.11 & 46.40 & 39.30 & 84.65 \\
\hline L.S.D $_{0.05}$ & 0.03 & 1.38 & 0.03 & 0.67 & 1.44 \\
\hline
\end{tabular}

Table 3. Mean values for expansion ratio as affected by the interaction between rice cultivars, temperature and time of popping in 2013 and 2014 seasons

\begin{tabular}{|c|c|c|c|c|c|c|c|c|c|}
\hline \multicolumn{2}{|c|}{ Treatments } & \multicolumn{4}{|c|}{2013} & \multicolumn{4}{|c|}{2014} \\
\hline \multirow{2}{*}{ Cultivars } & \multirow{2}{*}{$\begin{array}{c}\text { Temperature } \\
\left({ }^{0} \mathrm{C}\right)\end{array}$} & \multicolumn{4}{|c|}{ Time (sec) } & \multicolumn{4}{|c|}{ Time (sec) } \\
\hline & & 40 & 50 & 60 & 70 & 40 & 50 & 60 & 70 \\
\hline \multirow{5}{*}{ G. 178} & 220 & 6.56 & 7.68 & 8.16 & 7.99 & 6.18 & 6.80 & 7.66 & 7.31 \\
\hline & 230 & 6.96 & 8.37 & 8.76 & 8.59 & 6.52 & 7.36 & 8.20 & 8.02 \\
\hline & 240 & 7.82 & 8.69 & 9.35 & 9.12 & 7.40 & 8.15 & 8.76 & 8.51 \\
\hline & 250 & 7.93 & 9.13 & 9.77 & 9.45 & 7.65 & 8.52 & 9.15 & 8.79 \\
\hline & 260 & 8.30 & 9.47 & 10.65 & 9.62 & 8.07 & 8.92 & 10.11 & 9.15 \\
\hline \multirow{5}{*}{ G. 182} & 220 & 7.05 & 6.34 & 6.62 & 6.47 & 6.32 & 6.65 & 7.05 & 6.83 \\
\hline & 230 & 6.15 & 6.58 & 6.80 & 6.61 & 6.56 & 7.12 & 7.22 & 6.99 \\
\hline & 240 & 6.45 & 6.81 & 6.92 & 6.74 & 7.04 & 7.80 & 8.06 & 7.50 \\
\hline & 250 & 6.59 & 6.96 & 7.45 & 7.28 & 7.33 & 8.05 & 8.75 & 8.21 \\
\hline & 260 & 7.17 & 8.16 & 8.38 & 7.80 & 7.52 & 8.41 & 9.13 & 8.73 \\
\hline \multirow{5}{*}{ S. 102} & 220 & 6.11 & 6.36 & 6.72 & 6.61 & 6.41 & 6.91 & 7.33 & 7.08 \\
\hline & 230 & 6.34 & 6.69 & 6.86 & 6.78 & 6.70 & 7.33 & 7.65 & 7.45 \\
\hline & 240 & 6.60 & 6.93 & 7.35 & 7.19 & 7.04 & 7.80 & 8.15 & 8.06 \\
\hline & 250 & 7.12 & 7.35 & 7.79 & 7.55 & 7.25 & 7.95 & 8.61 & 8.34 \\
\hline & 260 & 7.46 & 7.65 & 8.55 & 8.12 & 7.62 & 8.33 & 8.85 & 8.49 \\
\hline \multirow{5}{*}{ S. 106} & 220 & 6.20 & 6.56 & 7.19 & 7.01 & 6.50 & 6.73 & 7.33 & 7.13 \\
\hline & 230 & 6.45 & 6.86 & 7.36 & 7.15 & 6.59 & 7.23 & 7.90 & 7.42 \\
\hline & 240 & 6.89 & 7.30 & 7.80 & 7.55 & 6.82 & 7.61 & 8.26 & 7.81 \\
\hline & 250 & 7.16 & 7.62 & 8.10 & 7.98 & 7.07 & 8.05 & 8.50 & 8.25 \\
\hline & 260 & 7.65 & 7.89 & 8.98 & 8.54 & 7.42 & 8.34 & 8.62 & 8.51 \\
\hline \multirow{5}{*}{$\begin{array}{l}\text { Egyptian } \\
\text { Yasmin }\end{array}$} & 220 & 6.49 & 6.84 & 7.28 & 7.10 & 6.63 & 7.05 & 7.42 & 7.25 \\
\hline & 230 & 6.79 & 7.17 & 7.58 & 7.36 & 7.06 & 7.41 & 7.75 & 7.50 \\
\hline & 240 & 7.52 & 7.77 & 8.16 & 7.92 & 7.34 & 7.89 & 7.93 & 8.10 \\
\hline & 250 & 8.00 & 8.25 & 8.69 & 8.48 & 7.65 & 8.85 & 9.38 & 9.15 \\
\hline & 260 & 8.19 & 8.45 & 9.89 & 9.35 & 7.85 & 9.12 & 9.56 & 9.31 \\
\hline \multicolumn{2}{|c|}{ L.S.D ${ }_{0.05}$} & \multicolumn{4}{|c|}{0.36} & \multicolumn{4}{|c|}{0.17} \\
\hline
\end{tabular}


Table 4. Mean values for weight after popping (g) as affected by the interaction between rice cultivars, temperature and time of popping in 2013 and 2014 seasons

\begin{tabular}{|c|c|c|c|c|c|c|c|c|c|}
\hline \multicolumn{2}{|c|}{ Treatments } & \multicolumn{4}{|c|}{2013} & \multicolumn{4}{|c|}{2014} \\
\hline \multirow{2}{*}{ Cultivars } & \multirow{2}{*}{$\begin{array}{c}\text { Temperature } \\
\text { ( } \mathrm{C} \text { C) }\end{array}$} & \multicolumn{4}{|c|}{ Time (sec) } & \multicolumn{4}{|c|}{ Time (sec) } \\
\hline & & 40 & 50 & 60 & 70 & 40 & 50 & 60 & 70 \\
\hline \multirow{5}{*}{ G. 178} & 220 & 45.10 & 45.34 & 45.61 & 45.40 & 44.50 & 44.82 & 45.15 & 45.07 \\
\hline & 230 & 45.29 & 45.58 & 45.92 & 45.65 & 45.15 & 45.41 & 45.66 & 45.49 \\
\hline & 240 & 45.75 & 45.94 & 46.32 & 46.01 & 45.56 & 45.60 & 45.83 & 45.67 \\
\hline & 250 & 46.16 & 46.28 & 46.83 & 46.35 & 45.81 & 45.89 & 46.15 & 46.10 \\
\hline & 260 & 46.48 & 46.60 & 47.27 & 46.93 & 46.07 & 46.31 & 46.83 & 46.55 \\
\hline \multirow{5}{*}{ G. 182} & 220 & 45.58 & 46.38 & 47.15 & 46.68 & 45.08 & 45.31 & 45.63 & 45.50 \\
\hline & 230 & 46.26 & 46.87 & 48.06 & 47.81 & 45.23 & 45.60 & 45.94 & 45.80 \\
\hline & 240 & 46.82 & 47.25 & 48.49 & 48.12 & 45.66 & 45.95 & 46.50 & 46.12 \\
\hline & 250 & 47.21 & 47.98 & 48.78 & 48.33 & 45.85 & 46.20 & 46.83 & 46.72 \\
\hline & 260 & 47.54 & 48.21 & 49.05 & 48.51 & 46.34 & 46.42 & 47.52 & 46.91 \\
\hline \multirow{5}{*}{ S. 102} & 220 & 45.25 & 45.67 & 46.39 & 46.25 & 44.70 & 45.05 & 45.56 & 45.20 \\
\hline & 230 & 45.80 & 46.01 & 46.80 & 46.47 & 44.93 & 45.50 & 45.91 & 45.77 \\
\hline & 240 & 46.34 & 46.67 & 47.28 & 46.85 & 45.36 & 46.07 & 46.34 & 46.22 \\
\hline & 250 & 46.73 & 47.09 & 47.78 & 47.15 & 45.62 & 46.21 & 46.90 & 46.56 \\
\hline & 260 & 47.22 & 48.17 & 48.60 & 47.99 & 45.89 & 46.73 & 47.38 & 46.93 \\
\hline \multirow{5}{*}{ S. 106} & 220 & 45.10 & 45.40 & 46.15 & 45.91 & 45.20 & 46.15 & 46.50 & 46.45 \\
\hline & 230 & 45.50 & 45.81 & 46.41 & 46.17 & 45.60 & 46.39 & 46.75 & 46.52 \\
\hline & 240 & 45.90 & 46.28 & 46.85 & 46.49 & 46.20 & 46.70 & 47.21 & 46.90 \\
\hline & 250 & 46.11 & 46.67 & 47.15 & 46.84 & 46.91 & 47.29 & 47.66 & 47.51 \\
\hline & 260 & 46.84 & 47.12 & 47.88 & 47.45 & 47.26 & 47.56 & 48.15 & 47.84 \\
\hline \multirow{5}{*}{$\begin{array}{l}\text { Egyptian } \\
\text { Yasmin }\end{array}$} & 220 & 45.06 & 45.30 & 45.88 & 45.56 & 45.35 & 45.91 & 46.32 & 46.12 \\
\hline & 230 & 45.34 & 45.66 & 46.15 & 45.85 & 46.20 & 46.51 & 46.79 & 46.70 \\
\hline & 240 & 46.05 & 46.25 & 46.55 & 46.15 & 46.35 & 46.65 & 47.07 & 46.83 \\
\hline & 250 & 46.17 & 46.53 & 46.83 & 46.46 & 46.71 & 46.92 & 47.36 & 47.13 \\
\hline & 260 & \multirow{2}{*}{\multicolumn{4}{|c|}{$\frac{46.89 \quad 47.44}{\mathbf{0 . 1 3}}$}} & \multirow{2}{*}{\multicolumn{4}{|c|}{$\frac{4 / .40}{0.15}$}} \\
\hline & & & & & & & & & \\
\hline
\end{tabular}

Table 5. Mean values for weight of popping (g) as affected by the interaction between rice cultivars, temperature and time of popping in 2013 and 2014 seasons

\begin{tabular}{|c|c|c|c|c|c|c|c|c|c|}
\hline \multicolumn{2}{|c|}{ Treatments } & \multicolumn{4}{|c|}{2013} & \multicolumn{4}{|c|}{2014} \\
\hline \multirow{2}{*}{ Cultivars } & \multirow{2}{*}{$\begin{array}{c}\text { Temperature } \\
\left({ }^{(} \mathrm{C}\right)\end{array}$} & \multicolumn{4}{|c|}{ Time (sec) } & \multicolumn{4}{|c|}{ Time (sec) } \\
\hline & & 40 & 50 & 60 & 70 & 40 & 50 & 60 & 70 \\
\hline \multirow{5}{*}{ G. 178} & 220 & 35.15 & 38.63 & 40.25 & 40.11 & 32.27 & 35.25 & 42.60 & 38.15 \\
\hline & 230 & 37.61 & 41.06 & 41.80 & 41.39 & 34.55 & 37.60 & 43.85 & 41.62 \\
\hline & 240 & 40.35 & 42.08 & 43.21 & 42.76 & 38.56 & 40.20 & 44.14 & 43.26 \\
\hline & 250 & 40.87 & 42.91 & 44.68 & 43.24 & 41.60 & 42.56 & 45.10 & 45.10 \\
\hline & 260 & 41.45 & 43.62 & 46.72 & 44.22 & 43.15 & 45.22 & 46.15 & 45.35 \\
\hline \multirow{5}{*}{ G. 182} & 220 & 32.15 & 30.68 & 33.34 & 33.03 & 30.15 & 33.36 & 35.54 & 35.10 \\
\hline & 230 & 32.65 & 33.26 & 36.56 & 37.13 & 31.86 & 35.27 & 40.15 & 38.16 \\
\hline & 240 & 38.67 & 39.61 & 40.81 & 39.27 & 35.20 & 39.25 & 41.26 & 40.50 \\
\hline & 250 & 40.08 & 40.37 & 41.12 & 40.84 & 41.20 & 40.20 & 41.69 & 40.90 \\
\hline & 260 & 40.34 & 40.72 & 41.78 & 41.20 & 41.65 & 41.54 & 42.95 & 42.10 \\
\hline \multirow{5}{*}{ S. 102} & 220 & 28.67 & 30.69 & 34.72 & 33.27 & 26.80 & 31.50 & 33.85 & 33.07 \\
\hline & 230 & 33.70 & 35.75 & 39.22 & 39.06 & 30.56 & 31.90 & 35.60 & 33.91 \\
\hline & 240 & 36.70 & 39.25 & 41.05 & 40.76 & 33.63 & 35.40 & 37.86 & 36.13 \\
\hline & 250 & 39.27 & 40.38 & 41.48 & 40.90 & 35.72 & 38.20 & 40.66 & 40.06 \\
\hline & 260 & 39.65 & 40.71 & 42.96 & 42.20 & 38.68 & 40.32 & 41.34 & 28.91 \\
\hline \multirow{5}{*}{ S. 106} & 220 & 30.85 & 35.18 & 38.80 & 37.72 & 32.19 & 32.65 & 35.31 & 33.60 \\
\hline & 230 & 35.10 & 37.18 & 40.25 & 39.76 & 34.20 & 35.71 & 38.55 & 35.90 \\
\hline & 240 & 38.12 & 40.15 & 41.45 & 40.85 & 35.62 & 39.15 & 40.08 & 39.50 \\
\hline & 250 & 40.26 & 40.78 & 42.03 & 41.40 & 39.20 & 41.08 & 41.94 & 41.36 \\
\hline & 260 & 40.69 & 41.14 & 43.21 & 41.69 & 41.63 & 42.85 & 45.12 & 44.17 \\
\hline \multirow{5}{*}{$\begin{array}{l}\text { Egyptian } \\
\text { Yasmin }\end{array}$} & 220 & 34.20 & 37.59 & 39.37 & 38.79 & 32.18 & 33.81 & 35.36 & 35.10 \\
\hline & 230 & 37.24 & 40.84 & 41.24 & 40.55 & 35.62 & 37.56 & 39.30 & 38.64 \\
\hline & 240 & 40.00 & 41.30 & 43.00 & 41.72 & 38.36 & 40.42 & 42.52 & 41.11 \\
\hline & 250 & 41.52 & 42.22 & 43.65 & 42.93 & 40.22 & 43.61 & 45.26 & 44.64 \\
\hline & 260 & 42.03 & 43.16 & 45.50 & 44.74 & 40.94 & 45.12 & 46.64 & 46.07 \\
\hline \multicolumn{2}{|c|}{ L.S.D $\mathbf{D . 0 5}$} & \multicolumn{4}{|c|}{0.25} & \multicolumn{4}{|c|}{3.36} \\
\hline
\end{tabular}


Table 6. Mean values for popping $\%$ as affected by the interaction between rice cultivars, temperature and time of popping in 2013 and 2014 seasons

\begin{tabular}{|c|c|c|c|c|c|c|c|c|c|}
\hline \multicolumn{2}{|c|}{ Treatments } & \multicolumn{4}{|c|}{2013} & \multicolumn{4}{|c|}{2014} \\
\hline \multirow{2}{*}{ Cultivars } & \multirow{2}{*}{$\begin{array}{c}\text { Temperature } \\
\left({ }^{\circ} \mathrm{C}\right)\end{array}$} & \multicolumn{4}{|c|}{ Time (sec) } & \multicolumn{4}{|c|}{ Time (sec) } \\
\hline & & 40 & 50 & 60 & 70 & 40 & 50 & 60 & 70 \\
\hline \multirow{5}{*}{ G. 178} & 220 & 77.94 & 85.20 & 88.26 & 88.36 & 72.52 & 78.65 & 94.35 & 84.64 \\
\hline & 230 & 83.04 & 90.09 & 91.02 & 90.65 & 76.52 & 82.80 & 96.04 & 91.49 \\
\hline & 240 & 88.20 & 91.60 & 93.29 & 92.94 & 84.64 & 88.16 & 96.31 & 94.72 \\
\hline & 250 & 88.53 & 92.73 & 95.41 & 93.30 & 90.81 & 92.74 & 97.72 & 97.82 \\
\hline & 260 & 89.17 & 93.61 & 98.83 & 94.22 & 93.66 & 97.65 & 98.55 & 97.42 \\
\hline \multirow{5}{*}{ G. 182} & 220 & 70.53 & 66.16 & 70.72 & 70.75 & 66.88 & 73.63 & 77.89 & 77.14 \\
\hline & 230 & 70.59 & 70.95 & 76.07 & 77.66 & 70.44 & 77.35 & 87.38 & 83.32 \\
\hline & 240 & 82.59 & 83.82 & 84.16 & 81.60 & 77.09 & 85.41 & 88.73 & 87.82 \\
\hline & 250 & 84.91 & 84.15 & 84.30 & 84.50 & 89.86 & 87.01 & 89.02 & 87.54 \\
\hline & 260 & 84.85 & 84.47 & 85.18 & 84.93 & 89.88 & 89.49 & 90.38 & 89.73 \\
\hline \multirow{5}{*}{ S. 102} & 220 & 63.35 & 67.19 & 74.84 & 71.93 & 59.96 & 69.91 & 74.30 & 73.16 \\
\hline & 230 & 73.58 & 77.71 & 83.81 & 84.06 & 68.01 & 70.11 & 77.54 & 74.10 \\
\hline & 240 & 79.20 & 84.10 & 86.83 & 86.99 & 74.14 & 76.83 & 81.70 & 78.17 \\
\hline & 250 & 84.03 & 85.75 & 86.82 & 86.75 & 78.30 & 82.67 & 86.70 & 86.05 \\
\hline & 260 & 83.96 & 84.52 & 88.39 & 87.94 & 84.28 & 86.28 & 87.25 & 61.64 \\
\hline \multirow{5}{*}{ S. 106} & 220 & 68.41 & 77.49 & 84.07 & 82.17 & 71.22 & 70.75 & 75.94 & 72.34 \\
\hline & 230 & 77.15 & 81.16 & 86.73 & 86.11 & 75.00 & 76.98 & 82.46 & 77.17 \\
\hline & 240 & 83.05 & 86.76 & 88.48 & 87.88 & 77.10 & 83.83 & 84.90 & 84.22 \\
\hline & 250 & 87.30 & 87.38 & 89.15 & 88.39 & 83.55 & 86.87 & 88.00 & 87.06 \\
\hline & 260 & 86.87 & 87.32 & 90.25 & 87.87 & 88.09 & 90.10 & 93.71 & 92.33 \\
\hline \multirow{5}{*}{$\begin{array}{c}\text { Egyptian } \\
\text { Yasmin }\end{array}$} & 220 & 75.90 & 82.97 & 85.81 & 85.15 & 70.96 & 73.64 & 76.34 & 76.11 \\
\hline & 230 & 82.13 & 89.46 & 89.37 & 88.45 & 77.10 & 80.76 & 83.99 & 82.74 \\
\hline & 240 & 86.86 & 89.29 & 92.37 & 90.40 & 82.76 & 86.65 & 90.33 & 87.79 \\
\hline & 250 & 89.92 & 90.74 & 93.22 & 92.41 & 86.11 & 92.95 & 95.57 & 94.72 \\
\hline & 260 & 90.21 & 92.05 & 95.91 & 95.18 & 86.97 & 95.19 & 97.74 & 96.92 \\
\hline \multicolumn{2}{|c|}{ L.S.D ${ }_{0.05}$} & \multicolumn{4}{|c|}{0.62} & \multicolumn{4}{|c|}{7.17} \\
\hline \multicolumn{6}{|c|}{$\begin{array}{l}\text { Popping percentage, for example, had an average over } \\
\text { the two seasons of } 8.02,0.46,4.39,5.13 \text { and } 9.34 \% \text { for } \\
\text { Giza 178, Giza } 182 \text {, Sakha } 102 \text {, Sakha } 106 \text { and } \\
\text { Yasmine, respectively. The results obtained from the } \\
\text { interaction revealed that increasing heating time from }\end{array}$} & $\begin{array}{l}\text { o-eat } \\
\text { add } \\
\text { nee } \\
\text { as } \mathrm{x}\end{array}$ & $\mathrm{cks}$ & $\begin{array}{l}\text { a gr } \\
\text { oducts, } \\
\text { sing to } \\
\text { efined }\end{array}$ & $\begin{array}{l}\text { market } \\
\text { nvenient } \\
\text { ds more } \\
\text { polished }\end{array}$ \\
\hline
\end{tabular}
40 to 60 seconds, at $260{ }^{\circ} \mathrm{C}$, may be of valuable in the case of the two varieties Giza 178 and Yasmine since both varieties showed high increase in expansion ratio accompanied with an increase popping \%. These two characters are important for the popping rice industry since they lead to an increase in yield. On the other hand, Giza 182 variety showed very low response to increasing heating time in popping \%. Hence it would be uneconomic to raise the heating time for 40 to 60 second.

\section{CONGLUSION}

Popping is a simple and inexpensive processing method which improves textural and sensory qualities of cereals and also there are minimum changes with respect to nutrient composition in the processed product. Traditionally, popped products are prepared only during few specific occasions. This type of home
The present study revealed variation between rice cultivars for the popping ability with variation in temperature and time of popping. That implies the need to optimize processing methods and factors which governs the popping characteristics of different cereal grains in order to get high popping yield, less unpopped kernels and higher expansion volume. The present study indicated that, Giza 178 rice cultivar with heating time of 60 second; at $260{ }^{\circ} \mathrm{C}$ gave the highest results for popping percentage in both seasons. Further studies are needed to assess micronutrients availability, dietary fiber content, protein and carbohydrate digestibility, to develop value added health foods to meet the community nutritional problems. There is also need for technology development for popping of different cereals to accomplish the target of achieving consumer satisfaction. 


\section{REFERENCES}

Abd El Salam, M.A.M. 2006. Chemical and technological studies on popped and puffed rice. M.Sc. Thesis, Fac. of Agric. Alex. Univ., Egypt.

Bhat Upadya VG, R.S. Bhat, V.V. Shenoy and P.M. Salimath .2008. Physico-chemical characterization of poppingspecial rice accessions. Karnataka J. Agric. Sci., 21 (2): 184-186.

Ceylan, M. and E. Karababa .2002. Comparison of sensory properties of popcorn from various types and sizes of kernel. J. Sci. Food Agric., 82: 127-133.

Delost-Lewis,K.; K. Lorenz, and K. Tribelhorn .1992. Puffing quality of experimental varieties of Prosco Millets. Cereal Chem., 69(4): 359-365.

Dofing, S.M.; M.A. Thomas-Compton, and J.S. Buck .1990. Genotype $\mathrm{x}$ popping method interactions for expansion volume in popcorn. Crop Sci., 30: 62-65.

Gokmen S. 2004. Effect of moisture content and popping method on popping characteristics of popcorn. J. Food Engineer., 65: 357-362.

Gomez,K.A. and A.A. Gomez .1984. Statistical Procedures for Agricultural Research. An International Rice Research Institute Book. John Willey and sons Inc., New York.

Henry, G., J.P. Schwartzberg, C. Wu, N. Amos and M. Joshua. 1995. Modelling deformation and flow during vapor-induced puffing. J. Food Engineer., 25(3): 357-362.

Hoke, K., J. Housova and M. Houska. 2005. Optimum conditions of rice puffing. Czech J. Food Sci, 23: 1-11.

Hoseney, R.C., K. Zeleznak, and A. Abd El-Rahman.1983. Mechanism of popcorn popping. J. Cereal Sci., 1: 43-52.

Hsieh, F., H.E. Huff, I.C. Peng and S.W. Marek (1989). Puffing of rice cakes as influenced by tempering and heating conditions. J. Food Sci., 54 (5): 1310-1312.

Huff, H.E., F. Hsieh and I.C. Peng. 1992. Rice cake production using long grain and medium grain brown rice. J. Food Sci., 57 (5): 1164-1167.
Joshi N.D., D. Mohapatra and D.C. Joshi. 2014. Varietal selection of some indica rice for production of puffed rice. Food and Bioprocess Tech., 7(1): 299-305.

Maisont, S. and W. Narkrugsa. 2010. Effects of salt, moisture content and microwave power on puffing qualities of puffed rice. Kasetsart J. Nat. Sci., 44 (2), 251-261.

Mirza, N., N. Sharma, S. Srivastava and A. Kuma. 2014. Variation in Popping Quality Related to Physical, Biochemical and Nutritional Properties of Finger Millet Genotypes. Proceedings of the National Acad. Sci., India Section B: Biological Sciences, 1-9.doi:10.1007/s400011014-0384-X.

Mohamed A.A., R.B. Ashman and A.W. Kirleis.1993. Pericarp thickness and other kernel physical characteristics relate to microwave popping quality of popcorn. J. Food Sci., 58: 342-346.

Murugesan, G. and K.R. Bhattacharya .1989. Rheological and hydration properties of popped rice. J. Texture studies, 20: 325-333.

SAS Institute. 1999. SAS System. Version 8. Statistical Analysis System Institute, Cary, NC, USA.

Shimoni E., E.M. Dirks and T.P. Labuza .2002. The relation between final popped volume of popcorn and thermalphysical parameters. Lebensmittel- Wissenschaft and Technology, 35: 93-98.

Song, A. and S.R. Eckhoff .1994. Optimum popping moisture content for popcorn kernels of different sizes. Cereal Chem, 71: 458-460.

Srinivas, T., and H.S.R. Desikachar. 1973. A simple inexpensive device for detecting and estimating cracks in intact paddy grains. J. Food. Sci. Tech., 10, 197.

Swarnakar, A.K., M. K. Devi, and S. K. Das .2014. Popping characteristics of paddy using microwave energy and optimization of process parameters. International Journal of Food Studies, Volume 3: 45-59.

Tian, Y., P. Buriak, and S. R. Eckhoff. 2001. Effect of hybrid and physical properties of individual popcorn kernels on expansion volume. Cereal Chem., 78: 578-582.

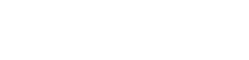

\section{تالثير الصنه والحرالرة وزمن الفشير عل صفلت الارز الفشار

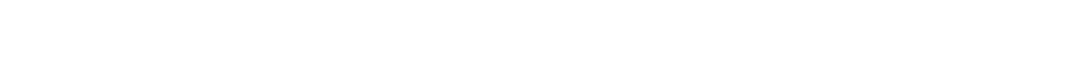

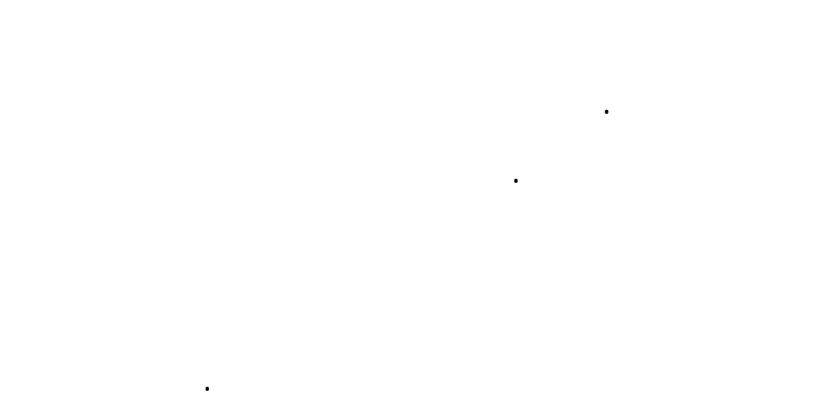
يهوف هذا البهث اله تحديد تأثير ظروف التصنبع والتى تتضمن درجة الحرارة والزمن على تمدد الارز

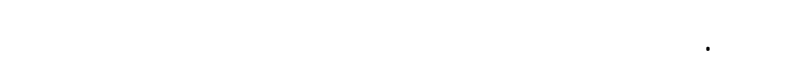
الاصنف فى مغظم الصفلت المدروسة وقد لظهر الصف وفون

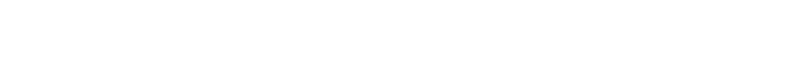
ونسبة القثير في كلا الموسمين. أدت زياة درجة الحرارة 